\title{
Materials Science Experiments under Microgravity - Spacelab 1
}

\section{A. Bewersdorff and B. Feuerbacher, Cologne}

(Institute for Space Simulation, DFVLR)

In November 1983, the European built Spacelab was carried into orbit by the American Space Shuttle and returned to Earth 10 days later. This flight presented a first opportunity to carry out research in the special environment of space, usscientific experiments. A total of 70 investigations were successfully performed, covering disciplines such as astronomy and solar physics, Earth observations, plasma physics, atmospheric physics, life- and materials sciences. Early results are documented in a special issue of Science 1 ).

In the area of materials sciences, 39 experiments were carried out, making use of the greatly reduced level of gravitational force aboard an orbiting spacecraft. Results were presented in November 1984 during a symposium at Schloss Elmau in Bavaria, which gave a comprehensive overview of the entire field $^{2}$ ). Here we report on just a few representative experiments.

Materials science experiments in space have given rise to wide public interest, but also to criticism in the scientific community, owing to the high cost involved and the widely heralded prospects of commercial benefits. While the commercial interest is developing slowly in Europe, in contrast to a much faster rise in the US ${ }^{3}$ ), the first set of experiments flown on Spacelab was directed mainly towards fundamental scientific goals. Earth gravity conditions. ing a laboratory specifically designed for

Gravity is an all-pervading force in the laboratory, but it is weak and usually of no particular concern to the experimenter. There are, however, situations - e.g. when density differences are present in a fluid medium - in which undesired gravity effects cannot be avoided: either the objective of an experiment may require temperature or concentration gradients which are imposed externally, or gradients appear spontaneously as a consequence of a natural process like solidification. Gravity then gives rise to convection or sedimentation of particulates, thus determining effectively the transport of heat and matter, as well as the equilibrium distributions of temperature and concentration. When $g$ is reduced by several orders of magnitude, other weaker forces or transport mechanisms are usually unveiled: for example, surface effects may become dominant. A system may thus evolve into a configuration or state not accessible under

The phenomenon of diffusion in liquids is one that lends itself as a candidate for space experimentation. Diffusion measurements require imposed gradients of concentration or temperature and are sensitive to even extremely weak convection currents in the liquid. An experiment by Frohberg et al2) was aimed at a precision measurement of diffusion in a liquid metal. Published data, particularly those referring to high

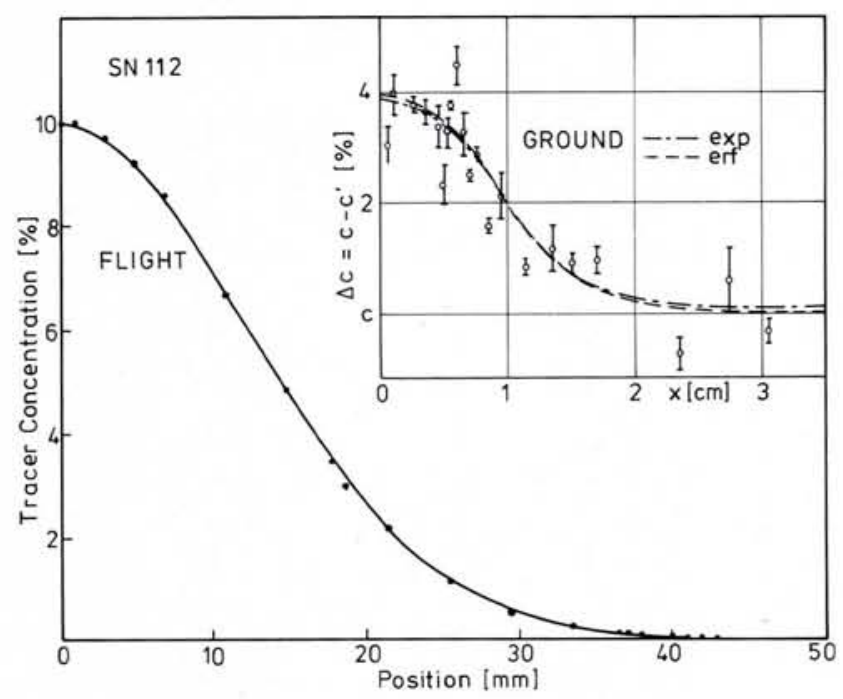

Fig. 1 - Self diffusion in liquid tin. The solid curve shows the concentration profile of $\mathrm{Sn}^{112}$ as a function of distance along the sample. Note the high measurement accuracy $(=0.5 \%)$. The inset illustrates typical ground results for the $S n$ system.

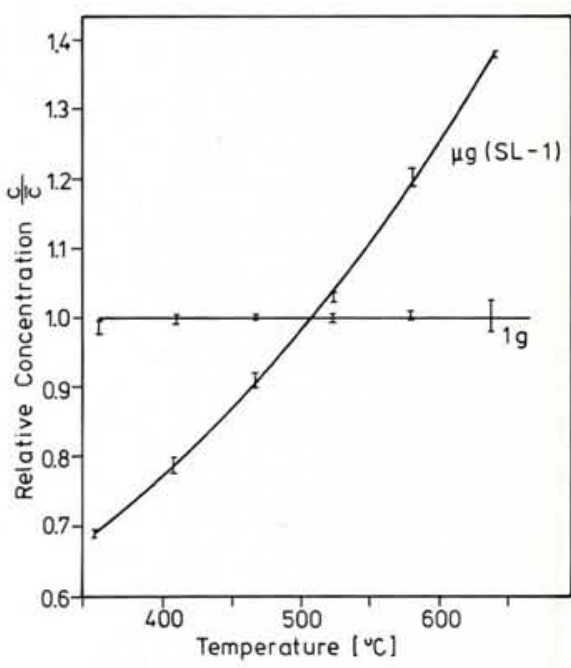

Fig. 2 - Thermomigration of cobalt in liquid tin. The curves show the relative concentration of cobalt as a function of temperature (or distance along the sample). The flight experiment $(\mu \mathrm{g})$ exhibits an enhancement of a factor two, while the ground data (Ig) are constant within the measurement accuracy.

temperatures, must be suspected of containing unknown contributions by convection and wall effects to the observed mass transport. As a consequence of this uncertainty, diffusion in liquids is not well understood and theories could not be subject to experimental scrutiny. The Spacelab experiment measured self diffusion in tin, a material well investigated in ground experiments. Two isotopes, ${ }^{112} \mathrm{Sn}$ and ${ }^{124} \mathrm{Sn}$, were applied to the ends of rods consisting of natural Sn. After diffusion at constant temperature, distributions as shown in Fig. 1 were obtained. The measurements yielded significantly lower diffusion coefficients than under normal gravity and moreover an improvement of accuracy by more than an order of magnitude compared to the best available ground-based data (inset Fig. 1). The higher accuracy allowed for the first time an isotope effect in liquid self diffusion to be reliably observed, when a difference of about $10 \%$ was found between the two coefficients.

Malmejac and Praizey ${ }^{2}$ ) observed the thermomigration of cobalt in liquid tin. Here the chemical separation of the components of a solution arising as a consequence of a thermal gradient was studied. A gradient of $200 \mathrm{deg} / \mathrm{cm}$ was maintained over a period of six hours across capillaries of $18 \mathrm{~mm}$ length. After the flight, the Co distribution was measured by activation analysis. The comparison of flight and ground results (Fig. 2) shows a surprising discrepancy: while a concentration difference of a factor 2 is found in the flight sample, the ground experiment shows a constant 
Fig. 3 - Ostwald ripening in a liquid dispersion alloy. The size distribution of $\mathrm{Pb}$ droplets in $\mathrm{Zn}$ is changed by about an order of magnitude after coarsening for $1 \mathrm{~h}$ in space. The relative width of the distribution remains constant. Such measurements are not accessible on the ground owing to sedimentation.

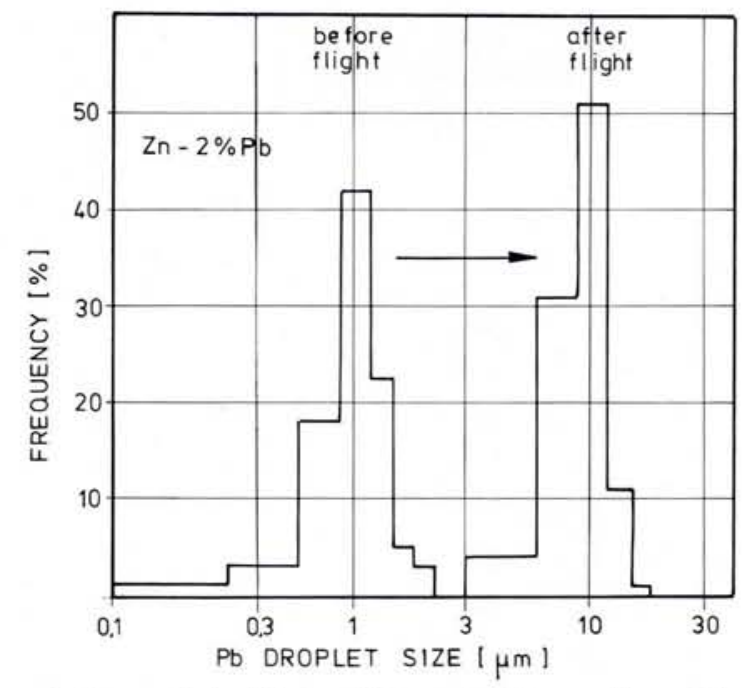

ticles (Ostwald ripening) in an alloy with a miscibility gap in the liquid phase was studied by Kneissl and Fischmeister ${ }^{2}$ ) for the $\mathrm{Zn}-\mathrm{Pb}$ system. Coarsening is a very common process in twocomponent mixtures and is caused by the size dependency of solubility. Quantitative measurements and tests of existing theories are normally hampered by sedimentation, which sets in when particles grow beyond the size of a few microns. Under microgravity, particle motions were negligible. The results obtained (Fig. 3) differ significantly from terrestrial measurements and allow us to define the range of validity of existing theories on the ripening process.

Solidification of various eutectic alloys was studied in two experiments ${ }^{2}$ ). With compositions solidifying into lamellar structures no difference was found between Earth and flight samples, whereas compositions yielding a fibrous structure of the minority phase showed a significantly coarser structure in the microgravity samples (Fig. 4). This is explained by a gravity-driven convection at the solidification front which on Earth cannot be controlled. It contributes effectively to the mass flow perpendicular to the growth direction and affects the solute distribution at the eutectic interface.

Various crystal growth experiments were performed during the first Spacelab flight. Results on semiconductor solidification by the floating zone method $^{2}$ ) demonstrated the influence of surface-induced Marangoni convection on dopant distribution (striations), which led to improved production methods on Earth. A particularly interesting case is the growth of insoluble crystals by a controlled precipitation reaction, which has been tested with two materials, $\mathrm{PnHPO}_{4}$ and $\mathrm{CaHPO}_{4} \cdot 2 \mathrm{H}_{2} \mathrm{O}^{2}$ ). These hydrophospha-

experiments indicated however a largescale phase separation even under microgravity conditions. which has been related to particle motion due to Marangoni-convection, a transport mechanism arising from spatial gradients in the interface tension induced by thermal gradients. An experiment of Ahlborn and Löhberg') confirmed this view, demonstrating the migration of precipitated particles along thermal gradients.

The coarsening of precipitated par-

Fig. 4 - Fibre density as a function of growth rate in the eutectic alloy InSb-NiSb. The flight sample $(\mu \mathrm{g})$ has a structure distinctly coarser than the ground sample (lg). The figure combines data from a Spacelab (SL-1) and a rocket (TEXUS) experiment.

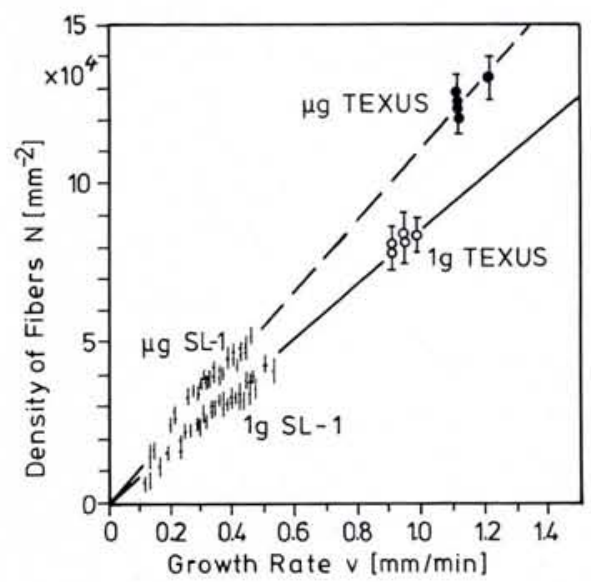

The Jülich Nuclear Research Centre (Kernforschungsanlage Jülich) is seeking for its Solid State Research Institute

(Institut für Festkörperforschung, IFF) a

\section{DIRECTOR}

as Head of one of its experimental institutes (previously the Institute for Superconductivity and Low-Temperature Physics).

The IFF comprises 7 experimental and 3 theoretical institutes, which carry out basic research in solid state physics, together with the necessary infrastructure.

The applicant should have outstanding knowledge and experience in experimental condensed matter physics and be capable of leading a scientific institute. He should be able to complement the present activities of the IFF by a new and promising field, preferably in the area of disorder phenomena or of phase boundaries and internal interfaces. The institute is expected to make use of German and international neutron sources (FRJ-2 Jülich; ILL Grenoble), and synchrotron sources (Hasylab, Hamburg; Bessy, Berlin; later the European Synchrotron Radiation Facility ESRF). Particular importance will be attached to a willingness to work with the other experimental and theoretical groups of the IFF. It is planned to arrange a joint appointment as a full professor (C-4) with one of the universities of North-Rhine-Westphalia.

Applications with curriculum vitae, list of publications and a short account of previous scientific activity should be sent by 15 th October 1985 to

VORSTAND DER KERNFORSCHUNGSANLAGE JÜLICH GmbH

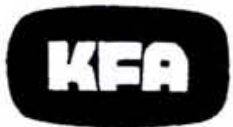

Postfach 1913

D-5170 Jülich

West Germany 
tes are representative of a class of incongruently melting or thermally decomposing materials that can be grown only at low temperatures. Under terrestrial conditions, growth is particularly difficult: either convection currents produce local supersaturation and thereby parasitic nucleation and growth bands, or the use of a gel eliminates convection but causes inclusions and other imperfections. The space samples demonstrated that good monocrystals can be grown by the method of coprecipitation. Here the reactants, initially separated, are allowed to diffuse into a nucleation chamber where the crystals are formed. Microgravity prevents natural convection and ensures mass transfer by diffusion only, resulting in monocrystals essentially free of dislocations. The experiment may ultimately open a way to producing a new class of crystal not accessible at present by ground methods with the perfection required for technical applications.

A similar problem is the growth of protein crystals for structural analysis. Here a minimum size of about $1 \mathrm{~mm}$ is needed to permit X-ray analysis at the resolution required. Such crystals are produced by the method of salting out from solution. This implies density gradients, which under gravity give rise to parasitic convection and cause multiseed formation. The experiment therefore yields small crystals that are useless. In space, diffusion-controlled nucleation and growth was achieved by Littke ${ }^{2}$ ) for lysozyme and -galactosidase (molecular weight 14307 and 465000 , respectively). The crystal sizes were significantly larger than those grown under Earth gravity conditions. The growth rates observed are considerably larger than expected and raise questions about our present understanding of crystallization of high molecular weight materials.

An experiment in fluid physics per-

EPS Divisions, Sections and Group
Astronomy and Astrophysics Division
Solar Physics Section
Atomic and Molecular Physics Division
Atomic Spectroscopy Section
Chemical Physics
Electronic and Atomic Collisions
Molecular Physics
Computational Physics Group
Condensed Matter Division
Liquids Section
Low Temperature Physics Section
Macromolecular Physics
Magnetism
Metal Physics
Semiconductors and Insulators
Surfaces and Interfaces
High Energy \& Particle Physics Division
Nuclear Physics Division
Optics Division
Plasma Physics Division
Quantum Electronics Division

formed by Padday ${ }^{2}$ ) was aimed at a quantitative measurement of intermolecular forces between liquids and solids. This was done by forming a liquid bridge between two specially shaped cylindrical disks. A liquid spreading on the lower disk develops a disjoining pressure, which is balanced by the capillary pressure of the liquid bridge. Microgravity allows large bridges to form of a shape close to the stability limit (a catenoid), such that small deviations from the ideal near the edges could be amplified and observed. Extremely low disjoining pressures were obtained $(0.4$ $0.009 \mathrm{~N} / \mathrm{m}^{2}$, to be compared with $10^{2}$ $\mathrm{N} / \mathrm{m}^{2}$ in Earth experiments), and wetting films of unexpected thickness $(0.2 \mathrm{~mm})$ shedding new light on our understanding of liquid-solid intermolecular forces.

The experiments described here show the variety of problems that can be accessed by the new tool of materials research under microgravity. New flight opportunities are available on rockets, Spacelab, space platforms or at a more distant future, a Space Station. The next of these opportunities will be the German Spacelab mission D1, scheduled for flight in October 1985. In view of these encouraging first results, scientists can be expected to continue to make effective use of this new research capability, expanding our fundamental understanding of matter and phenomena, and leading to novel products and processes.

\section{REFERENCES}

1. Science 255 (1984) pp. 163-232.

2. Proceedings of the 5th European Symposium "Materials Sciences and Microgravity: Results of Spacelab $1, \ldots$ ESA SP-222, 1984, available from ESA Scientific \& Technical Publications Branch, ESTEC Postbus 299, 2200 AG Noordwijk, The Netherlands (\$ 24)

3. Aviation Week \& Space Technology 120 (1984) 26, June pp. 40-201

Europhysics News is the official journal of the European Physical Society which comprises 29 National Socie ties, Academies and Group, over 4000 Individual Mem bers and 71 Associate Members. Governing bodies of EPS are the General Meeting, Council and an elected Executive Committee responsible for detailed policy. out Europe, organising and harmonising conferences and publications, improving physics education, encouraging physics applications, awarding scholarships to sponsored schools in Erice. EPS publishes in addition to sponsored schools in Erice. EPS publishes in addition to
EN, Europhysics Conference Abstracts, E. Ed. News and, in collaboration with The Institute of Physics (UK). the European Joumal of Physics. Individual Members receive EN free of charge (price to institutions: Sw.Fr. 90.-/a), rebates on the price of many publications and on conference fees. Annual EPS membership fee for 1986:Individual Members belonging to an EPS member society is: Sw.Fr. 44.-; independent members: Sw.Fr. 132.; members of a Collaborating Society, e.g. the American Physical Society: Sw.Fr. 55.- (\$26).

\section{Society News}

\section{New Associate Members}

ACEC - Atelier de Constructions Electriques de Charleroi

B - 6000 Charleroi

Bell Telephone Manufacturing Co. Francis Wellesplein 1

B - 2018 Antwerpen

GTE ATEA N.V.

Atealaan

Industrie Park Klein Gent

B - 2410 Herentals

LNETI - Laboratorio Nacional de Engenharia e Technologia Industrial

Azinhaga dos Lameiros à Estrada do Paço do Lumiar P - 1699 Lisboa Codex

\section{IMEC - Interuniversitair Micro-}

Elektronica Centrum VZW,

Kapeldreef 75

B-3030 Leuven

\section{CMD General Conferences}

The Chairman of the Condensed Matter Division, has pointed out that the Berlin Conference was «in place of» not «joined to" the traditional March Physikertagung of the German Physical Society as indicated in the May issue of Europhysics News. It is now established that the EPS CMD General Conference is held instead of the national conference of the host country.

\section{Corrections}

We apologise for citing in the June issue as President of The Institute of Physics, the former instead of the present incumbent who is:

Sir Alec Merrrison

Also for misspelling the name of the new editor of the European Journal of Physics:

Professor J. Pisut.

\section{Editor: E.N. Shaw \\ Meetings Compilation: W.S. Newman \\ Editorial Board: \\ K. Appert, A. Baratoff, B. Jacrot, \\ G.R. Macleod, M. Mayor, J. Muller}

Editorial and Advertising Office at the EPS Secretariat

Address: EUROPEAN PHYSICAL SOCIETY P.O. Box 69 CH-1213 Petit-Lancy 2 Switzerland

Telephone: Geneva (22) 931130

Telex: $\mathbf{4 2 3} \mathbf{4 5 5}$ dema ch

Cables: europhys genève

Printed by: Pfirter frères sa $\mathrm{CH}-1213$ Petit-Lancy/Switzerland 